\title{
Transalpina
}

TRANSALPINA Études italiennes

\section{2 | 2019}

Traductions, adaptations, réceptions de l'œuvre de Giovanni Verga

\section{Gianni Oliva, D'Annunzio. Tra le più moderne vicende}

\section{Luca Bani}

\section{(2) OpenEdition \\ Journals}

Edizione digitale

URL: http://journals.openedition.org/transalpina/525

DOI: $10.4000 /$ transalpina.525

ISSN: 2534-5184

Editore

Presses universitaires de Caen

\section{Edizione cartacea}

Data di pubblicazione: 1 novembre 2019

Paginazione: 178-180

ISBN: 978-2-84133-944-0

ISSN: 1278-334X

Notizia bibliografica digitale

Luca Bani, «Gianni Oliva, D'Annunzio. Tra le più moderne vicende », Transalpina [Online], 22 | 2019, online dal 01 novembre 2019, consultato il 19 novembre 2020. URL : http://journals.openedition.org/ transalpina/525; DOI : https://doi.org/10.4000/transalpina.525 


\section{Gianni Oliva, D’Annunzio. Tra le più moderne vicende, Milano, Bruno Mondadori, 2017, 203 p.}

Gianni Oliva ha una lunga tradizione di studi dannunziani. Nel corso degli anni ha curato l'edizione dell'opera omnia per la Newton Compton (1995), ha studiato i contatti di d'Annunzio (e di Pascoli) con le riviste dell'estetismo fiorentino (Minerva Italica 1979 e Marsilio 2002), ha analizzato le novelle di Terra vergine (Solfanelli 1994), ha presentato il carteggio tra d'Annunzio e Masciantonio (Ianieri 2001) e, soprattutto, ha curato l'edizione delle lettere ai Treves (Garzanti 1999).

Ora, con questa nuova monografia, Oliva si addentra nella fitta rete di relazioni tra d'Annunzio e il mondo moderno approfondendo differenti aspetti della vicenda biografica e artistica dannunziana ed evidenziando la biunivocità di questo rapporto. Se infatti la modernità ha costituito la linfa e il motore di tante suggestioni dannunziane, che nella velocità, nel movimento e nelle metamorfosi incessanti a cui è sottoposto il mondo contemporaneo ha trovato infiniti spunti narrativi, dall'altro è risaputo come il Pescarese abbia a sua volta influito sulla modernità, assecondandone il cammino ma cercando, al contempo, di imprimerle il sigillo della bellezza per cercare di salvarla da quella dimensione di puro utilitarismo e di riduzione dell'essere umano a mero soggetto consumatore che è uno dei temi forti di tanta narrativa otto-novecentesca.

Dopo la Premessa, nella quale viene richiamato il colloquio D'Annunzio et la modernité svoltosi a Caen nel 2008 e viene chiarito come, nella prospettiva dannunziana, proprio «la bellezza [...] deve ispirare l'andamento delle cose, anche delle più complesse, adattandosi al dinamismo contemporaneo, persino alla macchina [...]» (p. 2), il volume si articola in dieci capitoli e in due Appendici finali. Queste ultime, in realtà, sono un po' estravaganti rispetto al soggetto principale dell'opera, perché la prima (Carteggi e studi recenti del « laboratorio » di Chieti) è una rassegna dello stato di pubblicazione dei carteggi dannunziani e un aggiornamento sui lavori e sulle ricerche in corso facenti capo al centro ASAM (Archivio Scrittori Abruzzesi e Meridionali) e al dottorato in Lingua e letteratura delle regioni d'Italia, entrambi con sede presso l'università di Chieti; mentre la seconda (Una lettera-prefazione per una «dolce rimatrice») è la trascrizione di una conversazione tenuta da Oliva a Morrovalle, in provincia di Macerata, che riguarda la poetessa Lalla Vicoli Nada e la sua raccolta intitolata Versi, uscita nel 1905 con una lettera di d'Annunzio posta in esergo come prefazione.

Il corpo centrale del testo è quindi suddiviso nelle seguenti sezioni : Ironia e leggerezza del "giovin signore»; La macchina, il volo; Faville di guerra; Di un amico avverso; Il più arguto degli editori. Le lettere ai Treves; 
Il moderno comunicatore. Le conversazioni con i giornalisti; Il linguaggio dello sport; Il romanzo veneziano e la tradizione musicale italiana; La guerra per la Fedra rivisitata e Malinconie per Eleonora: annotazioni in margine.

Il primo capitolo tratta di un aspetto insolito e poco conosciuto di d'Annunzio, la sua propensione all'ironia nell'esprimere giudizi sui più diversi argomenti - soprattutto quando si occupa di critica letteraria - e all'autoironia, rintracciabile ad esempio nei molteplici nom de plume adottati dall'autore in particolare per i suoi scritti giornalistici; mentre nel secondo Oliva esamina la relazione tra d'Annunzio e gli aspetti che forse maggiormente lo affascinavano dell'avventura della modernità, cioè la macchina, la velocità e il volo, per poi passare nel terzo al rapporto tra d'Annunzio e la Prima guerra mondiale attraverso la scrittura delle Faville, e nel quarto ai legame tra d'Annunzio e Cesare De Lollis. Oliva torna poi, nel quinto capitolo, a un tema che, come si è ricordato, è già stato oggetto delle sue ricerche, ossia i carteggi con i Treves, per dedicarsi in quello successivo a vagliare i collegamenti tra d'Annunzio, i giornalisti - con l'analisi delle interviste dedicate ai temi della poesia, del romanzo del teatro e del cinema e il giornalismo in generale, considerato uno strumento fondamentale e di «grande potenzialità » (p. 91) per la propagazione del messaggio del Vate. Ci sono poi un capitolo sul Linguaggio dello sport, nel quale l'Autore analizza, concentrandosi in particolare sull'ambito sportivo, l'« esterofilia linguistica di d'Annunzio [...] che sarebbe appannaggio della sua vocazione allo sperimentalismo e alla modernità» (p. 120), seguito da una parte dedicata alla Fedra di Umberto Bozzini e alla contiguità di quest'autore e della sua opera con d'Annunzio e con la sua tragedia, a partire dalla coincidenza di date per cui lo stesso 10 aprile 1909 contemporaneamente veniva pubblicata la prima e rappresentata per la prima volta la seconda. Chiude il volume il capitolo intitolato Malinconie per Eleonora: annotazioni in margine a un epistolario nel quale Oliva fornisce il catalogo delle lettere scambiate dai due amanti ancora esistenti, analizza lo stile epistolare dannunziano e ripercorre i momenti salienti della relazione.

Una segnalazione particolare merita il capitolo ottavo, intitolato Il romanzo veneziano e la tradizione musicale italiana. I rapporti tra il Pescarese e l'arte musicale sono stati oggetto di studio almeno sin dalla celebre monografia di Alfredo Casella pubblicata nel 1939 a Milano per i tipi dei fratelli Bocca. Altri studi si sono poi aggiunti nel corso dei decenni, come il volume di Adriana Guarnieri Corrazzol, Sensualità senza carne: la musica nella vita e nell'opera di d'Annunzio, uscito nel 1990 per il Mulino, o la miscellanea di studi pubblicati in occasione del Convegno internazionale di Gardone del 1988. Il contributo di Oliva parte dal Fuoco, dall'atteggiamento di d'Annunzio verso Wagner e dalla dimestichezza che 
il protagonista Stelio Effrena, e quindi l'Autore stesso, sembra avere quando si sofferma su riflessioni e considerazioni musicali. Oliva ripercorre quindi le alterne fortune del wagnerismo nelle opere di d'Annunzio, a partire dagli articoli sul Caso Wagner del 1893 sino all'evocazione del compositore nel Trionfo della morte e alla sua messa in discussione, appunto, nel Fuoco, romanzo nel quale al «gigante» di Lipsia cominciano a venir contrapposti sia autori italiani, da Palestrina a Monteverdi e alla Camerata dei Bardi, sia tedeschi, da Bach a Beethoven, verso i quali Wagner avrebbe un indiscutibile debito. L'obiettivo principale di d'Annunzio, tuttavia, è senza dubbio quello di creare un'alternativa "mediterranea" all'imperio della musica tedesca, e «tale volontà di riscatto comincia con Il Fuoco, si protrarrà per lunghi anni ancora, accrescendosi di elementi complessi, fino all'elogio incondizionato di Ildebrando Pizzetti, autore della partitura musicale della Fedra dannunziana, che aveva mostrato di aver compiuto il pellegrinaggio al sepolcro dei Frari e meditato a lungo sulla tomba di Monteverdi» (p. 142).

Luca BANI

Gabriele d'Annunzio, Alcyone, edizione critica a cura di Pietro Gibellini, commento di Giulia Belletti, Sara Campardo ed Enrica Gambin, scheda metrica di Gianfranca Lavezzi, Venezia, Marsilio (Letteratura universale / ESPERIA), 2018, 925 p.

Alcyone è senz'ombra di dubbio la silloge dannunziana più letta, studiata, commentata e, non ultimo, amata, perché in essa il poeta pescarese riesce come mai prima - e soprattutto dopo - a dare il meglio di sé, amalgamando esemplarmente la raffinatezza linguistica di cui è maestro con l'ispirazione poetica e con l'interiorizzazione della materia liricizzata: il risultato finale è un'essenza di versi distillati con tale maestria da risultare del tutto naturali, le parole perfette per descrivere una sia pur breve parentesi di assoluta totalità. Non è un caso, dunque, se anche solo a partire dal 2000 si possono contare ben dieci edizioni (e riedizioni) della raccolta, una ogni due anni, alle quali se ne possono aggiungere una parziale e bilingue italiano / francese (De l'Alcyone et autres poemes, choix, tradution de l'italian et notes de Muriel Gallot, preface de Giuseppe Conte, Paris, Orphée-La Différence, 2013) e un'altra, in questo caso completa, sempre bilingue italiano/ tedesco (Alcyone, ubersetzt von Ernst-Jurgen Dreyer und Geraldine Gabor unter Mitarbeit von Hans Krieger, mit einem Anhang versehen von Geraldine Gabor und einem Nachwort von Ernst-Jurgen Dreyer, Berlin, Elfenbein, 2013). 